\title{
In-vitro cytotoxicity of biosynthesized gold nanoparticles against thyroid cancer cell lines
}

\author{
Yuan-Mei Li*, Yuan-Yuan Wang and Bo-Ning Cheng \\ Department of Endocrinology, Xixi Hospital of Hangzhou CITY, Hangzhou 310023, Zhejiang, China
}

${ }^{*}$ For correspondence: Email: liyuanmeiym@hotmail.com

Sent for review: 13 July 2016

Revised accepted: 7 June 2017

\begin{abstract}
Purpose: To undertake the biosynthesis of gold nanoparticles (AuNPs) using Shorea tumbuggaia bark extract and to study their in-vitro cytotoxicity in thyroid cancer (SW579) cell lines.

Methods: AuNPs were prepared by adding $10 \mathrm{~mL}$ of Shorea tumbuggaia extract to $5 \mathrm{~mL}$ of $2 \times 10^{-3} \mathrm{M}$ of chloroauric acid $\left(\mathrm{HAuCl}_{4}\right)$ and stirred at room temperature for about $20 \mathrm{~min}$. The AuNPs were evaluated by $x$-ray diffraction (XRD), Fourier transform infrared spectroscopy (FT-IR), ultraviolet-visible (UV-Vis) spectroscopy and transmission electron microscopy (TEM). They were also assessed for cytotoxicity against SW579 cells using 3-(4,5-dimethylthiazol-2-yl)-2,5-diphenyltetrazolium bromide (MTT) assay.

Results: The reaction of Shorea tumbuggaia extract with $\mathrm{HAuCl}_{4}$ led to a change in the color of the reaction solution to ruby red after $20 \mathrm{~min}$, indicating the formation of AuNPs. The results of various instrumental tests, including XRD, TEM, UV-vis spectroscopy and FT-IR spectroscopy confirmed the formation of AuNPs. TEM images showed spherical NPs with a mean particle size of $20 \mathrm{nM}$. Further, invitro cytotoxicity results indicated concentration-dependent cytotoxicity against SW579 cell lines.

Conclusions: A simple, green and low-cost method for the preparation of AuNPs using Shorea tumbuggaia extract has been achieved. The AuNPs exert cytotoxic activity against SW579 cell lines.
\end{abstract}

Keywords: Shorea tumbuggaia, Polyphenols, Gold nanoparticles, Cytotoxicity, Thyroid cancer cell

\begin{abstract}
Tropical Journal of Pharmaceutical Research is indexed by Science Citation Index (SciSearch), Scopus, International Pharmaceutical Abstract, Chemical Abstracts, Embase, Index Copernicus, EBSCO, African Index Medicus, JournalSeek, Journal Citation Reports/Science Edition, Directory of Open Access Journals (DOAJ), African Journal Online, Bioline International, Open-J-Gate and Pharmacy Abstracts
\end{abstract}

\section{INTRODUCTION}

Nanoparticles (NPs) have been regarded as important materials in the fast growing field of nanoscience and nanotechnology. Due to their extraordinary size-dependent features, they have a wide scope of applications in many natural and human-related activities. Several physical and chemical methods are currently available for the effective production of NPs; however, due to their expensive and dangerous nature for bulk production, these synthetic approaches are less preferred for industrial scale production of NPs. Microbes, plant extracts and biomolecules could be considered alternative source materials for traditional chemical and physical methods for the biosynthesis of NPs [1-3].

Several studies have investigated the synthesis of gold $(\mathrm{Au})$ and silver (Ag) NPs by green methods using plant extracts, yeast, bacteria, fungi and honey [4]. The larvicidal activity of noble metal NPs synthesized from Eclipta prostrata leaf extracts against vectors of malaria and filariasis was demonstrated by Rajakumar and Abdul Rahuman [5]. Similarly, antimicrobial AgNPs and AuNPs active against clinically isolated pathogens have also been reported [6].

In the present work, we demonstrated a simple 
and green synthetic method for AuNP preparation in water using Shorea tumbuggaia bark extract polyphenols. Further, we have also studied the in-vitro cytotoxicity of the biosynthesized AuNPs against thyroid cancer cell lines (SW579).

\section{EXPERIMENTAL}

\section{Materials}

Chloroauric acid $\left(\mathrm{HAuCl}_{4}\right)$, dimethyl sulfoxide (DMSO), the 3-(4,5-dimethylthiazol-2-yl)-2,5diphenyltetrazolium bromide (MTT) reagent and other solvents were purchased from SigmaAldrich Chemical Co. All experiments were performed using double distilled water as a medium.

\section{Preparation of Shorea tumbuggaia leaf extract}

The leaves of the Shorea tumbuggaia plant were collected from plants located nearby to Xixi Hospital in Hangzhou city in the month of August 2015 followed by drying under sunlight, and it was authenticated by Wei Zhang, a taxonomist at Institute of Botany, a herbarium in Beijing (PE). The voucher specimen of the plant leaf was kept for reference at the herbarium of Institute of Botany, Beijing (PE) with voucher no. 153.

The dried bark was then ground into a powder using a pestle and mortar. The bark extract was prepared by adding $5 \mathrm{~g}$ of fine dried powder of Shorea tumbuggaia bark to $50 \mathrm{~mL}$ of deionized water and then boiling for about $30 \mathrm{~min}$ in a temperature-controlled water bath. The mixture was cooled to room temperature and filtered to obtain a clear extract solution.

\section{Synthesis of silver nanoparticles}

About $10 \mathrm{~mL}$ of Shorea tumbuggaia extract was added to $5 \mathrm{~mL}$ of $2 \times 10^{-3} \mathrm{M} \mathrm{HAuCl}_{4}$ and the subsequent reaction mixture was stirred at room temperature for about $20 \mathrm{~min}$. The formation of AuNPs was indicated by the change in the color of the reaction solution to ruby red.

\section{Cell culture}

The SW579 cell lines were maintained in Dulbecco's modified Eagle's medium (DMEM) supplied with $100 \mu \mathrm{M}$ streptomycin, $100 \mu \mathrm{M}$ penicillin and $10 \%$ fetal calf serum (FCS). The cells were then maintained at $37^{\circ} \mathrm{C}$ with $5 \% \mathrm{CO}_{2}$ in a humidified cell incubator.

\section{Cell viability by MTT assay}

Cell viability studies were performed following a previously reported procedure [7]. Briefly, the cells were seeded in 96-well plates at a density of 1,500 cells per well and left overnight to attach to the walls. Then, the cells were exposed to various concentrations of AuNPs (0.05, 0.1 and $0.5 \mathrm{mM}$ ) for about $24 \mathrm{~h}$ at $37{ }^{\circ} \mathrm{C}$ under $5 \% \mathrm{CO}_{2}$ atmospheric conditions. MTT solution was then added to the cells at a final concentration of 0.5 $\mathrm{mg} / \mathrm{mL}$ and the cells were further incubated for 4 h. The optical absorbance of the cell suspensions was recorded at $560 \mathrm{nM}$ using an Elisa reader. The $\mathrm{IC}_{50}$ value for AuNPs was also determined.

\section{Characterization of AuNPs}

The formation of the AuNPs was spectroscopically recorded using a Shimadzu 2400 ultraviolet-visible (UV-Vis) spectrophotometer at room temperature. The morphology and size of the Shorea tumbuggaia extract-mediated AuNPs was studied using a JEOL JEM 2100 high-resolution-transmission electron microscope (HR-TEM). Samples were prepared for analysis by placing a drop of Au nano colloid onto the copper grid surface and drying under vacuum.

A selected area electron diffraction (SAED) pattern was simultaneously recorded. The X-ray diffraction (XRD) pattern of the AuNPs was recorded using a Bruker D8 Advance diffractometer over $10^{\circ}$ to $80^{\circ}$ with a scan run of $4 \% \mathrm{~min}$, step size of $0.02^{\circ}$ and $\mathrm{Cu} \mathrm{Ka}$ radiation of $\lambda=1.54 \mathrm{~A}^{\circ}$. In addition, the dynamic light scattering (DLS) and zeta potential of the biosynthesized AuNPs was analyzed using a nanoparticle analyzer (SZ-100; Horiba Scientific Nanoparticci). The Ag nano colloid was diluted 10 times and used for DLS and surface charge measurements. The surface capping oxidized polyphenols of the prepared AuNPs was obtained by Fourier transform infrared (FT-IR) spectroscopy (JASCO). A purified AuNP powder was studied using FT-IR.

\section{Statistical analysis}

All experiments were performed in triplicate for each treatment. Absolute values of each assay were transformed into control percentages. The data are the mean of three separate experiments while error bars are used to represent standard error of the mean. Differences between groups were determined by 
Student's t-test and statistical significance was set at $p<0.05$.

\section{RESULTS}

The conversion of $\mathrm{Au}^{+3}$ to $\mathrm{Au}^{0}$ was confirmed initially by measuring the UV-visible spectrum of the Au nanocolloid by reaction time. The UVvisible optical absorption spectrum of the Shorea tumbuggaia-mediated AuNPs is represented in Figure 1 . The existence of a well-defined absorption band at $535 \mathrm{nM}$, shown by the synthesized colloid, further indicated the formation of AuNPs.

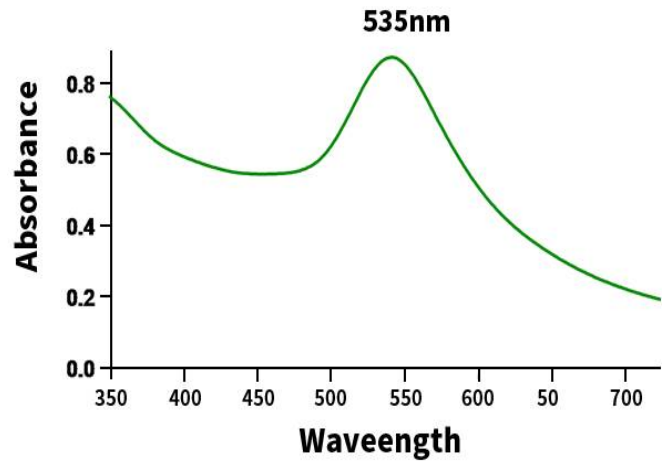

Figure 1: Ultraviolet-visible absorption spectrum of gold nanoparticles (AuNPs)

The crystal structure of the AuNPs synthesized using Shorea tumbuggaia extract was analyzed using XRD. The XRD analysis of the AuNPs is shown in Figure 2, and confirms that the synthesized NPs had a face cantered cubic (FCC) structure, with corresponding diffractions peaks at $2 \theta$ values of $38.2^{\circ}, 44.48^{\circ}, 64.7^{\circ}$ and $77.7^{\circ}$. The characteristic indexing planes of AuNPs were (1 111$),\left(\begin{array}{lll}2 & 0 & 0\end{array}\right),\left(\begin{array}{lll}2 & 2 & 0\end{array}\right)$ and (3 111$)$ respectively; this further confirmed the crystalline structure of the AuNPs (JCPDS File No.870720).

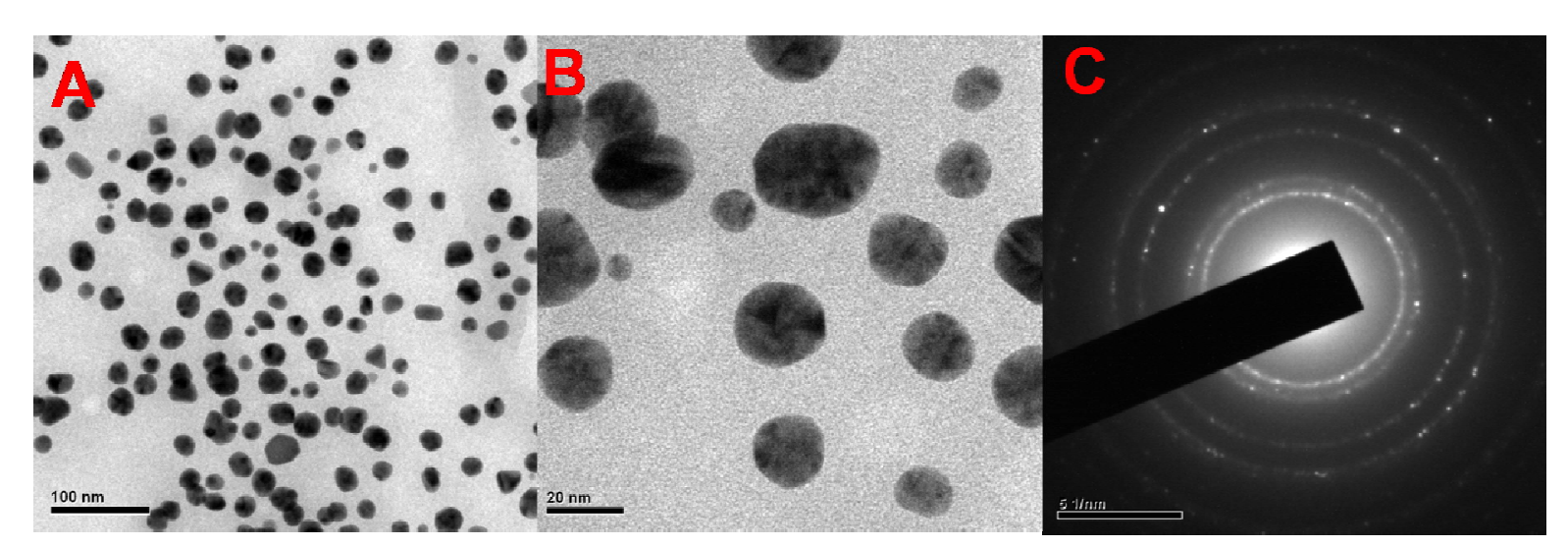

Figure 3: Transmission electron microscopy (TEM) images (A, B) and selected area electron diffraction (SAED) pattern of biosynthesized AuNPs
Figure $3 A$ and $B$ show the TEM images of the biofabricated AuNPs. The TEM microscopic images showed that AuNPs are spherical in shape and polydispersed in nature, with an average particle size of $20 \mathrm{~nm}$. Further, the SAED pattern of AuNPs, shown in Figure 3C, confirmed the crystalline structure of the synthesized AuNPs with well-distinguished diffraction spots.

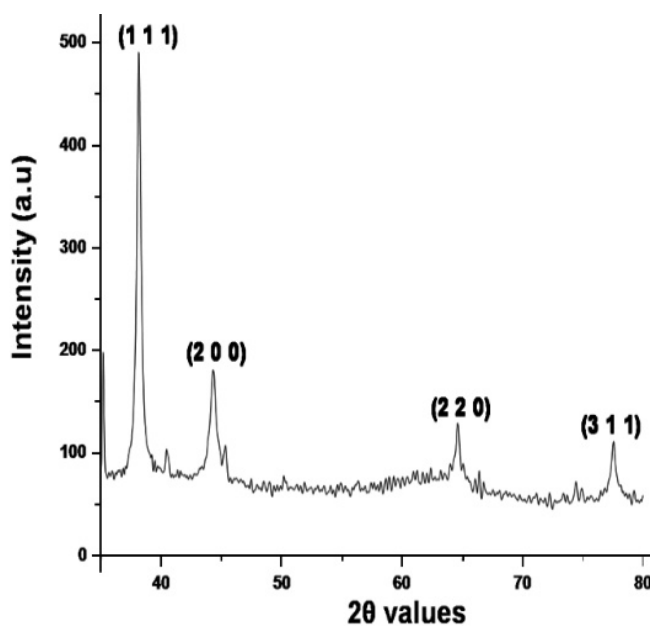

Figure 2: X-ray diffraction (XRD) patterns of biosynthesized AuNPs

Figure 4 shows the FTIR spectrum of the green synthesized AuNPs. Vibrational stretching bands at 3,395 and $1,383 \mathrm{~cm}^{-1}$ were observed, related to the stretching of hydroxyl and tertiary alcoholic groups. The bands present at 1,712, 1,615 and $1,055 \mathrm{~cm}^{-1}$ can be ascribed to ketonic, carboxylic $-\mathrm{C}=\mathrm{O}$ and $-\mathrm{C}-\mathrm{O}-\mathrm{C}$ vibrational stretching, respectively. The size distribution and zeta potential of the synthesized AuNPs is shown in Figure 5A. The mean particle size of the NPs was found to be $20 \mathrm{nM}$. The zeta potential measurement on the AuNPs showed the negative surface charge of the synthesized NPs and was found to be $-55 \mathrm{mV}$ (Figure 5B). 


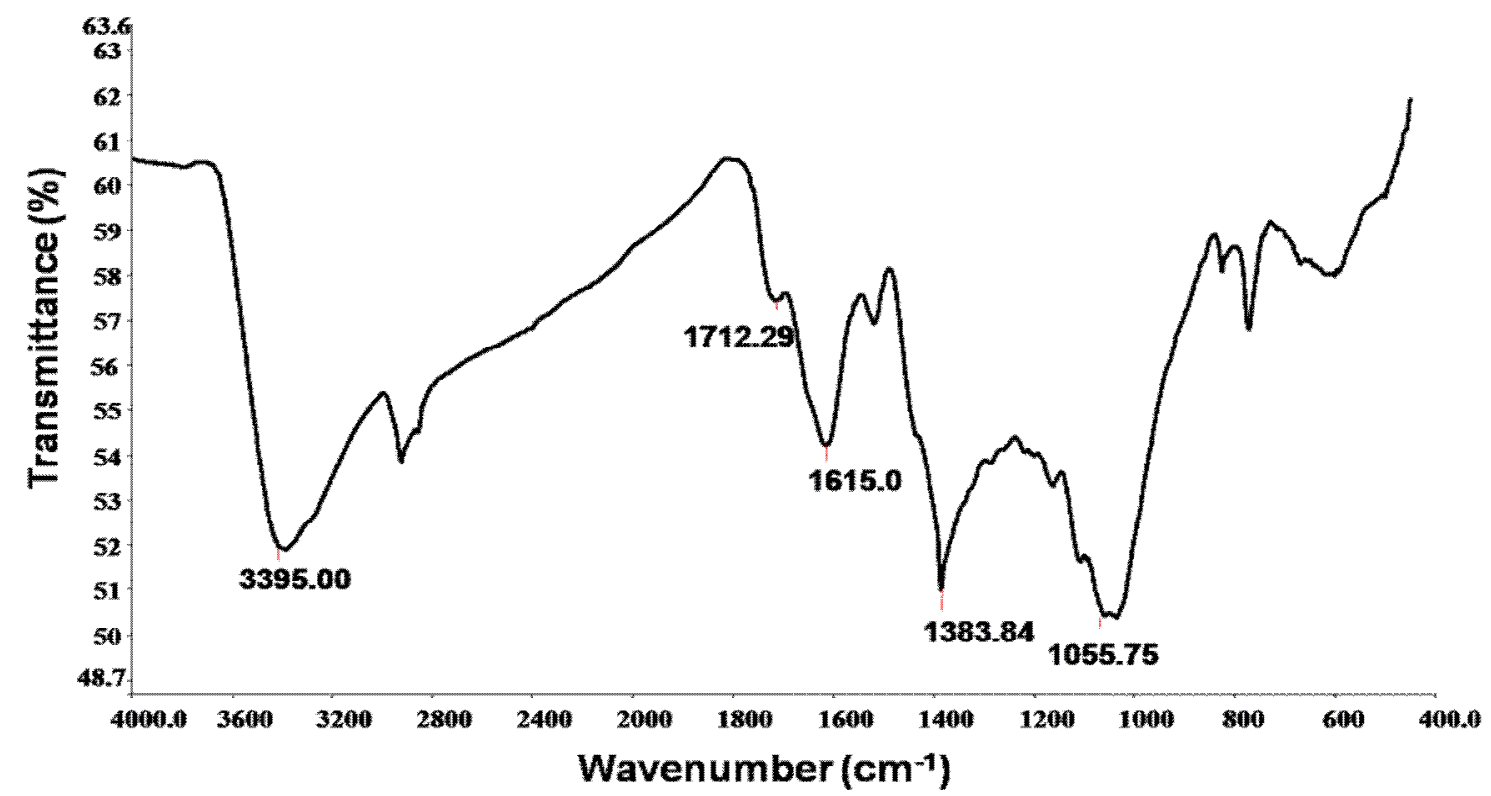

Figure 4: Fourier transform infrared spectroscopy (FT-IR) spectrum of biosynthesized AuNPs

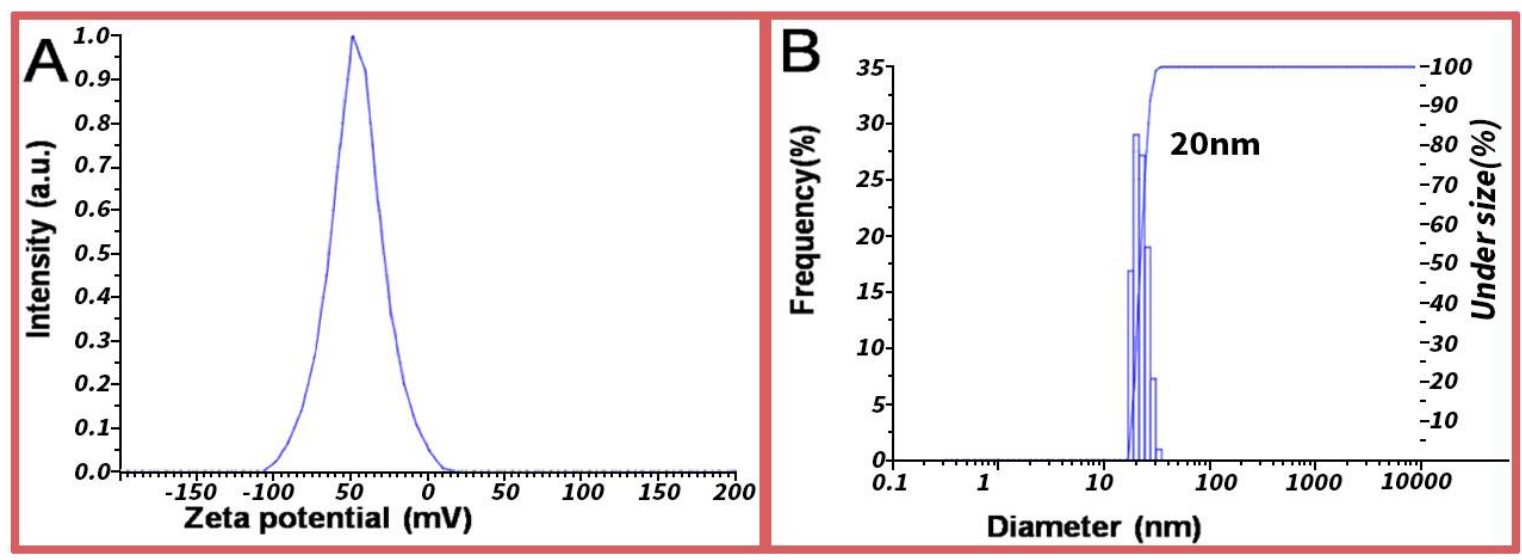

Figure 5: Dynamic light scattering (DLS) analysis (A) and zeta potential (B) of AuNPs synthesized using Shorea tumbuggaia extract

\section{In-vitro cytotoxicity}

The AuNPs were successfully prepared to investigate their cytotoxicity against thyroid cancer cell lines (SW579). The concentration effect of AuNPs on the cell viability of SW579 cell lines was studied by treatment with various concentrations of AuNPs $(0.05, \quad 0.1$ and 0.5 $\mathrm{mM})$. An in-vitro cytotoxicity assay on SW579 showed the toxic nature of AuNPs (Figure 5). The percentage cell viabilities were $88 \%$ and 38 $\%$ when treated with AuNPs at concentrations of 0.05 and $0.5 \mathrm{mM}$, respectively. It is confirmed that the cell viability percentage is decreased with increase in the concentration of AuNPs.

\section{DISCUSSION}

Shorea tumbuggaia extract containing naturally occurring polyphenols was used as a reducing and capping agent for the preparation of AuNPs. The $-\mathrm{OH}$ groups of polyphenols of Shorea tumbuggaia extract play a significant role in the conversion of $\mathrm{Au}^{+3}$ to $\mathrm{Au}^{0}$ and subsequent stabilization of the readily formed Au nuclei [8]. The formation of AuNPs was confirmed visually by the change of color of the reaction solution from colorless to ruby red. The intense ruby red color of the synthesized AuNPs is due to the surface plasmon resonance (SPR) phenomenon of the noble metal NPs, caused by the interaction of surface conduction electrons of AuNPs with electromagnetic radiation [9]. The existence of an SPR band at $535 \mathrm{nM}$ in the UV-visible optical absorption spectrum further confirmed the $\mathrm{Au}^{+3}$ reduction. 
The formation of AuNPs was also confirmed by TEM and XRD analysis. The presence of XRD peaks characteristic of AuNPs and related indexing planes showed the crystalline structure of the synthesized NPs. The mean particle size obtained for the AuNPs was $20 \mathrm{nM}$, which is also supported by DLS analysis results. This result for the size of the AuNPs is in good agreement with the SPR absorption band of the AuNPs; however, it has been previously reported that the SPR absorption band of gold and silver NPs depends on their morphology and size [10].

The formation of spherical and smaller AuNPs is highly advantageous because of their applications in bioimaging and drug delivery. For instance, smaller spherical NPs can easily pass through the cell membrane, while larger NPs cannot. Moreover, the existence of biomolecular constituents on the surface of the synthesized NPs may increase the biocompatibility of the synthesized NPs [10].

The FT-IR spectral studies revealed stabilization of the synthesized AuNPs with plant biomolecules. The existence of vibrational bands characteristic of the hydroxyl and carboxylic groups in the FT-IR spectrum revealed stabilization of oxidized polyphenols onto the AuNPs surfaces. The presence of a vibrational stretching band characteristic of the ketonic group at $1,712 \mathrm{~cm}^{-1}$ further indicated the key role of $-\mathrm{OH}$ groups of Shorea tumbuggaia extract polyphenols in $\mathrm{Au}^{+3}$ reduction [8]. The consecutively formed oxidized polyphenols will cap the readily formed $\mathrm{Au}$ nuclei and thus stabilize them. It has been extensively reported that the $-\mathrm{OH}$ groups of plant polyphenols will donate electrons for $\mathrm{Au}^{+3}$ reduction and subsequently convert into their quinone forms. The resulting oxidized polyphenols will cap the formed $\mathrm{Au}$ nuclei. These results are also supported by the negative surface zeta potential of AuNPs, which later generates repulsive forces among the NPs resulting in the reduced agglomeration of NPs [11].

An MTT assay was conducted to investigate the cytotoxicity of the synthesized AuNPs. Several reports have already shown the cytotoxicity of metal NPs against different cancer cell lines [12]; however, there are no studies available on concentration-dependent toxicity against SW579 cell lines using AuNPs synthesized with Shorea tumbuggaia extract. The cytotoxicity results of the synthesized AuNPs indicated that the biosynthesized NPs showed considerable cytotoxicity towards SW579 cell lines, with cytotoxicity increasing with increasing NP concentration. From these results, it can be concluded that the toxicity of the green synthesized AuNPs is dose-dependent. The toxicity dependence on AuNP concentration against human lung cancer cell lines has been reported previously [10]; thus, AuNPs synthesized with the present method using Shorea tumbuggaia extract could act as antithyroid cancer agent through their cytotoxic mechanisms.

\section{CONCLUSION}

A low cost, green approach for the preparation of AuNPs using Shorea tumbuggaia extract is demonstrated in this work. Zeta potential and FTIR results indicate that there is capping of oxidized polyphenols onto the AuNP surfaces. Furthermore, Shorea tumbuggaia extract polyphenol-coated AuNPs exhibit concentrationdependent cytotoxicity against SW579 cell lines. Hence, the synthesized AuNPs may find application in the synthesis of anti-cancer drugs in the future.

\section{DECLARATIONS}

\section{Acknowledgement}

The authors gratefully acknowledge the support of Xixi Hospital, Hangzhou City for this research.

\section{Conflict of Interest}

No conflict of interest associated with this work.

\section{Contribution of Authors}

The authors declare that this work was done by the authors named in this article and all liabilities pertaining to claims relating to the content of this article will be borne by them.

\section{Open Access}

This is an Open Access article that uses a funding model which does not charge readers or their institutions for access and distributed under the terms of the Creative Commons Attribution License (http://creativecommons.org/licenses/by/ 4.0) and the Budapest Open Access Initiative (http://www.budapestopenaccessinitiative.org/rea d), which permit unrestricted use, distribution, and reproduction in any medium, provided the original work is properly credited.

\section{REFERENCES}

1. Sastry M, Ahmad A, Khan MI, Kumar R; Niemeyer M, Eds. Microbial nanoparticle production. Nano-biotech- 
nology, Wiley-VCH, Weinheim 2004; pp 126-135.

2. Bhattacharya $D$, Rajinder $G$. Nanotechnology and potential of microorganisms. Crit Rev Biotechnol. Crit. Rev Biotechnol 2005; 25: 199-204.

3. Mohanpuria P, Rana NK, Yadav SK. Biosynthesis of nanoparticles: technological concepts and future applications. J. Nanopart. Res. 2008; 10: 507-517.

4. Kharissova OV, Rasika Dias HV, Kharisov BI, Perez BO and Jimenez Perez VM. The greener synthesis of nanoparticles. Trends in Biotechnol 2013; 31: 240-248.

5. Rajakumar G, Abdul Rahuman A. Larvicidal activity of synthesized silver nanoparticles using Eclipta prostrata leaf extract against filariasis and malaria vectors. Acta Tropica 2011; 118: 196-203.

6. MubarakAli $D$, Thajuddin $N$, Jeganathanb $K$, Gunasekaran M. Plant extract mediated synthesis of silver and gold nanoparticles and its antibacterial activity against clinically isolated pathogens. Colloids Surf. 2011; 85: 360-365.

7. Wang CJ, Yang D, Luo YW. RhoBTB2 (DBC2) functions as a multifunctional tumor suppressor in thyroid cancer cells via mitochondrial apoptotic pathway. Int J Clin Exp Med 2015; 8: 5954-5958.
8. Mohan Kumar K, Badal Kumar M, Kiran Kumar HA, Sireesh Babu M. Green synthesis of size controllable gold nanoparticles. Spectrochim. Acta Mol. Biomol. Spectrosc. 2013; 116: 539-545.

9. Prashant KJ, Huang X, El-Sayed IH, El-Sayed MA. Review of Some Interesting Surface Plasmon Resonance-enhanced Properties of Noble Metal Nanoparticles and Their Applications to Biosystems. Plasmonics. 2007; 2: 107-118.

10. Sireesh babu M, Badal Kumar M, Kiran Kumar A. Environment friendly approach for size controllable synthesis of biocompatible Silver nanoparticles using diastase. Environ. Toxicol. Pharmacol. 2017; 49: 131 136.

11. Hemali P, Pooja M, Sumitra C. Green synthesis of silver nanoparticles from marigold flower and its synergistic antimicrobial potential. Arabian Journal of Chemistry. 2015; 8: 732-741.

12. Johnston HJ, Hutchison G, Christensen FM, Peters S, Hankin $S$ and Stone $V$. A review of the in vivo and in vitro toxicity of silver and gold particulates: particle attributes and biological mechanisms responsible for the observed toxicity. Crit. Rev. Toxicol. 2010; 40: 328-346. 